\title{
Additively Manufactured Flexible and Stretchable Antenna Systems for Wearable Applications
}

\author{
Atif Shamim \\ Electrical Engineering Program \\ King Abdullah University of \\ Science and Technology \\ (KAUST) \\ Thuwal, Saudi Arabia \\ atif.shamim@kaust.edu.sa
}

\author{
Mohammad Vaseem \\ Electrical Engineering Program \\ King Abdullah University of \\ Science and Technology \\ (KAUST) \\ Thuwal, Saudi Arabia \\ mohammad.vaseem@kaust.edu.sa
}

\author{
An Sizhe \\ Electrical Engineering Program \\ King Abdullah University of \\ Science and Technology \\ (KAUST) \\ Thuwal, Saudi Arabia \\ an.sizhe@kaust.edu.sa
}

\author{
Muhammad Fahad Farooqui \\ Electrical Engineering Program \\ King Abdullah University of \\ Science and Technology \\ (KAUST) \\ Thuwal, Saudi Arabia \\ muhamaad.farooqui@kaust.edu.sa
}

\begin{abstract}
Emerging applications such as Internet of things (IoT) and wearable sensors require new kind of electronics that can be bent, stretched, worn, washed, etc. These electronics must be extremely low cost, to the extent that they become disposable. The flexibility and low-cost aspects can be addressed by using additive manufacturing techniques, such as inkjet and screen printing on light-weight and flexible substrates like paper or plastics. However, the best solution for wearable electronics is to print them directly on textiles. Though, the driving electronics are still predominantly realized in standard CMOS platforms but all the remaining parts of these systems, such as sensors, antennas, interconnects, etc, that are large and expensive to realize on CMOS, can be printed. These could be integrated with CMOS chips to demonstrate system level wearable examples. This paper will show some examples of such flexible and stretchable components and systems that have been realized through additive manufacturing. Performance issues under flexed and stretched conditions are discussed. The promising results of these designs indicate that the day when electronics can be printed like newspapers and magazines through roll-to-roll and reel-to-reel printing is not far away.
\end{abstract}

Keywords-flexible electronics, additive manufacturing, inkjet printing, wearable electronics.

\section{INTRODUCTION}

According to predictive estimates, twenty to thirty billion devices will be connected to the internet by year 2020, to form what we know as IoT today [1]. An important aspect is that these devices may be mounted on non-planar or curved surfaces and may also be required to be worn by humans as part of wearable sensors. Thus, conformability, flexibility and strechability will be an important design and fabrication aspect for these connected devices. Another critical issue is the low-cost requirement for these IoT devices, to the extent that they can be produced in billions and be disposable in some cases. To cater for low cost and flexibility requirements, new manufacturing techniques must be considered.

Additive manufacturing such as inkjet printing is attractive for electronics manufacturing as it is completely digital, does not require masks and deposits materials only where it is required, unlike the traditional subtractive fabrication methods. This can facilitate low cost, high-throughput fabrication of electronics on unconventional flexible substrates such as paper or plastics or even textiles, which can be highly suitable for emerging applications such as wireless sensors for IoT or other flexible and wearable devices, etc.

Most of the wearable electronics devices require wireless connectivity to communicate the sensors' data or for wireless powering. This means radio frequency (RF) components such as transmission lines, filters, antennas must also be realized on these flexible mediums. It is well known that RF components get effected the most with the changes in the substrate or environment around them. It is thus very important to study the effects of flexibility, stretching and washing on such components. A number of inkjet printed antennas [2-4] and sensors [5-6] have been demonstrated on flexible substrates. However, performance issues while being flexed or stretched have mostly been overlooked in the previous works.

In this paper, we present RF components and systems, realized through additive manufacturing that can be flexed, stretched and worn in some cases. The performance is assessed when these components and systems are bent or stretched. We also show direct printing on textile (stretchable sports T-shirt) through new stretchable dielectric and metallic inks and compare the performance with standard (nonstretchable ink). It is also shown that the T-shirt, with printed electronics, can be washed multiple times but still maintains decent RF performance.

\section{FLEXIBLE RF COMPONENTS \& SYSTEMS}

\section{A. Inkjet Printed Flexible Antenna}

A multi-band antenna, inkjet printed with silver nanoparticles based ink on Teslin paper, is shown in Fig. $1(\mathrm{a}, \mathrm{b})$ [7-8]. This antenna is composed of four branch lines on the top side (antenna part) and three L-shaped slots on the bottom side (ground plane). This arrangement helps in generating multiple bands. The heating (sintering of the ink) does not make it rigid or hard (as is observed in case of a polymer coated paper), and it maintains its flexibility. Complete RF characterization has been done in flat and flexed conditions and it sis observed that the performance is deteriorated a bit in bent conditions, but to acceptable levels.

\section{B. Inkjet Printed Wearable Antenna Systems}

Two examples of wearable antennas are shown in Fig. 2, [9-10]. In the first example (shown in Fig. 2 (a)), where a complete WiFi based localization system has been 
demonstrated directly on textile (cotton T-Shirt material), an inverted $\mathrm{F}$ antenna has been realized through inkjet printing.

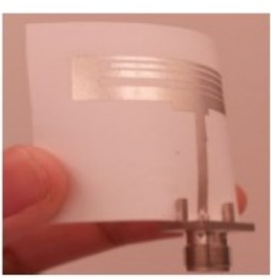

(a)

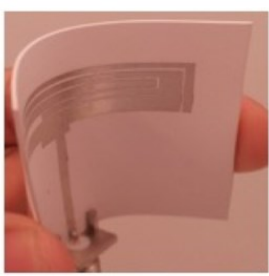

(b)
Fig. 1. Flexible Antennas (a) Bent outward, (b) Bent inward

The antenna is directly matched to the WiFi chip and provides a communication range of around $55 \mathrm{~m}$ and a localization accuracy of approximately $15 \mathrm{~m}$ while worn on a human body. In the second example (shown in Fig. 2 (b)), a loop antenna has been inkjet printed on a flexible Kapton tape which is part of a smart bandage to monitor chronic wounds condition. The sensors have been printed on a common bandage, while the wireless part (on Kapton tape) has been attached to this bandage. The bandage, while worn on a human body, can communicate sensors data to approximately $60 \mathrm{~m}$.

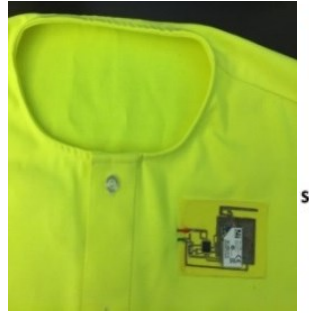

(a)

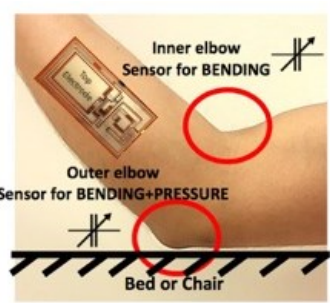

(b)
Fig. 2. Wearable antenna systems, (a) WiFi Antenna system for localization (b) Bluethooth based smart bandage

\section{STRETChIBLE AND WASHABALE RF COMPONENTS}

In order to assess the RF performance on stretchable materials, a microstrip filter is designed and screen printed directly on textile (a sports $\mathrm{T}$-shirt) using a stretchable dielectric ink DuPont PE773 and a stretchable Ag conductive ink DuPont PE873 [11], and is shown in Fig. 3. The dielectric ink is used as an adhesion layer as well as a protection layer to avoid shorting of signal and ground conductors. For comparison, the same filter is also printed using a nonstretchable conductor paste, DuPont PE819.
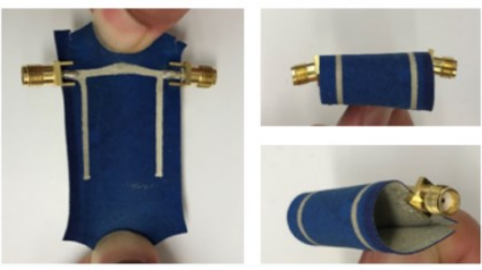

Fig. 3. Stretching and flexing of the filter

The results are shown in Fig. 4 where it can be clearly seen that the filter can be stretched up to $25 \%$ with small deterioration in performance. However, the non-stretchable ink based filter develops cracks and results in zero transmission (Fig. 4(b)). This cracking can be clearly seen in Fig. 5.

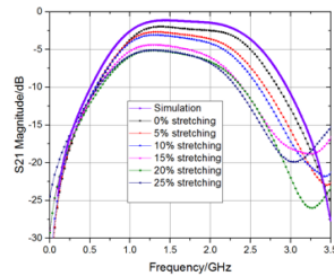

(a)

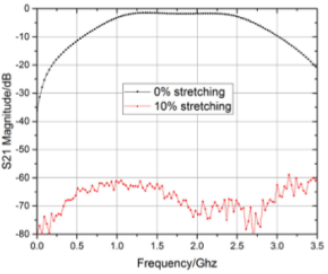

(b)
Fig. 4. Filter transmission $\left(\mathrm{S}_{21}\right)$, (a) Stretchable Ink (b) Non-strecthable Ink

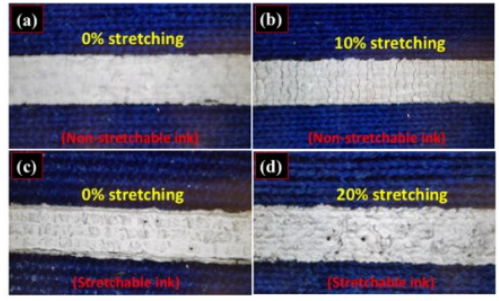

(a)

(b)

Fig. 5. Microscopic Images (a) Stretchable Ink (b) Non-strecthable Ink

\section{REFERENCES}

[1] Available online: http://spectrum.iee.org/techtalk/telecom/internet/popular-internet-of-things-forecast-of-50-billiondevices-by-2020-is-outdated," IEEE Spectrum, Aug 2016.

[2] B. S. Cook, A. Shamim, "Inkjet Printing of Novel Wideband and High Gain Antennas on Low- Cost Paper Substrate," IEEE Transactions on Antennas and Propagation, vol. 60, pp.4148 - 4156, 2012.

[3] H. Abutarboush, A. Shamim, "Paper-Based Inkjet Printed Tri-Band USlot Monopole Antenna for Wireless Applications," IEEE Antennas and Wireless Propagation Letters, vol. 11, pp.1234-1237, 2012.

[4] L. Yang, A. Rida and M. Tentzeris, "RFID Tag and RF Structures on a Paper Substrate Using Inkjet-Printing Technology", IEEE Transactions on Microwave Theory and Techniques, pp. 2894-2901, 2007.

[5] M. F. Farooqui, C. Claudel, and A. Shamim, " An Inkjet Printed Buoyant 3-D Lagrangian Sensor for Real Time Flood Monitoring", IEEE Transactions on Antennas \& Propagation, vol. 62, pp.3354-3359, 2014.

[6] A. Quddious, S. Yang, M. M. Khan, F. A. Tahir, A. Shamim, K. Salama and H. Cheema, "Disposable, Paper-Based, Inkjet-Printed Humidity and H2S Gas Sensor for Passive Sensing Applications", MDPI Sensors, 2016

[7] H. Abutarboush, M. Farooqui and A. Shamim, "Inkjet-Printed Wideband Antenna on Resin-Coated Paper Substrate for Curved Wireless Devices," in IEEE Antennas and Wireless Propagation Letters, pp. 20-23, 2016.

[8] H. F. Abutarboush and A. Shamim, "Paper-Based Inkjet-Printed TriBand U-Slot Monopole Antenna for Wireless Applications," in IEEE Antennas and Wireless Propagation Letters, vol. 11, no., pp. 12341237, 2012.

[9] B. Krykpayev, M. Farooqui, R. Bilal, M. Vaseem, A. Shamim, "A wearable tracking device inkjet-printed on textile," Microelectronics Journal, Volume 65, Pages 40-48, 2017.

[10] M. Farooqui and A. Shamim, "Low Cost Inkjet Printed Smart Bandage for Wireless Monitoring of Chronic Wounds", Nature Scientific Reports, 6:28949, 2016.

[11] Availableonline:http://www.dupont.com/content/dam/dupont/productsand-services/electronic-and-electricalmaterials/documents/prodlib/PE873.pdf 